\title{
IdeAs
}

Idées d'Amériques

$6 \mid 2015$

Migrer dans les Amériques

\section{Entre brèches et héritages: Mobilité académique des élèves ingénieurs mexicains dans la seconde moitié du vingtième siècle}

Entre brechas y herencias: La movilidad académica de los ingenieros mexicanos en la segunda mitad del siglo XX

Gaps and heritages: Mexican engineer students' academic mobility in the second half of the 20th century

\section{Etienne Gérard et Rocio Grediaga}

\section{CpenEdition}

\section{Journals}

Édition électronique

URL : https://journals.openedition.org/ideas/1112

DOI : $10.4000 /$ ideas. 1112

ISSN : $1950-5701$

Éditeur

Institut des Amériques

Référence électronique

Etienne Gérard et Rocio Grediaga, « Entre brèches et héritages : Mobilité académique des élèves ingénieurs mexicains dans la seconde moitié du vingtième siècle », IdeAs [En ligne], 6 | 2015, mis en ligne le 02 décembre 2015, consulté le 20 octobre 2022. URL : http://journals.openedition.org/ideas/ 1112 ; DOI : https://doi.org/10.4000/ideas. 1112

Ce document a été généré automatiquement le 20 octobre 2022

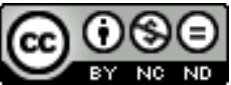

Creative Commons - Attribution - Pas d'Utilisation Commerciale - Pas de Modification 4.0 International - CC BY-NC-ND 4.0

https://creativecommons.org/licenses/by-nc-nd/4.0/ 


\section{Entre brèches et héritages : Mobilité académique des élèves ingénieurs mexicains dans la seconde moitié du vingtième siècle}

Entre brechas y herencias: La movilidad académica de los ingenieros mexicanos en la segunda mitad del siglo XX

Gaps and heritages: Mexican engineer students' academic mobility in the second half of the 20th century

Etienne Gérard et Rocio Grediaga

\section{Introduction}

1 Depuis ses origines, la science base son développement sur les experts des différents domaines, où qu'ils se trouvent. Aux 12è et 13è siècles déjà, les étudiants se déplaçaient là où résidaient ceux qui pouvaient leur enseigner ; cette dynamique s'est intensifiée et a changé de modalités du 19è siècle à nos jours (Charle, Schriewer et Wagner, 2006 ; Moulinier, 2012). Les mobilités étudiantes actuelles sont en particulier inscrites dans des politiques d'internationalisation de l'enseignement supérieur mises en œuvre par une part croissante d'Etats, du "Nord" comme du "Sud", engagés dans l'attraction des étudiants internationaux dans le cadre d'une libéralisation du commerce des services de l'éducation et d'une nouvelle gestion publique de l'enseignement supérieur (Vinokur et Sigman, 2010). Cependant, comme le soulignent Mazzella et Eyebiyi, «il faut maintenir dans le champ d'analyse de l'internationalisation de l'enseignement supérieur la force historique des échanges binationaux, qui configurent encore les champs scientifiques nationaux : la mobilité internationale des étudiants continue de suivre les voies traditionnelles de coopération qui se sont construites progressivement au cours de l'histoire [...]» (Mazzella et Eyebiyi, 2014 : 7). 
2 Dans le prolongement de cette "historicité" des liens scientifiques entre communautés et espaces scientifiques nationaux et étrangers, ce travail s'interroge sur l'héritage dont l'ingénierie (tecnología) mexicaine est le produit. Plus précisément, dans quelle mesure celle-ci s'est-elle construite grâce aux formations à l'étranger des ingénieurs mexicains?

3 Pareille interrogation nécessite d'identifier en priorité les mobilités pour études des élèves ingénieurs : dans quelles proportions les ingénieurs mexicains se sont-ils formés à l'étranger? Dans quel pays? La mobilité pour études a-t-elle concerné toutes les disciplines de l'ingénierie? Après avoir retracé brièvement l'histoire de l'ingénierie mexicaine, telle sera la première série de questions auxquelles nous apporterons ici des réponses pour rendre compte de l'impact des formations à l'étranger sur la structuration de l'ingénierie mexicaine. Nous serons conduits à identifier les pays et des institutions dans lesquels les générations successives d'ingénieurs mexicains se sont formés ; pays et institutions que nous dénommerons "pôles de formation ».

En second lieu : comment expliquer la mobilité des élèves ingénieurs vers l'étranger? Dans le cas du Mexique, trois principaux facteurs à l'origine de ces mobilités sont couramment avancés dans la littérature pour en expliquer l'origine et l'accroissement : les politiques d'internationalisation, les politiques de coopération universitaire de ce pays avec ses partenaires étrangers, ou encore l'extension du marché international de la formation, impulsée en particulier par les politiques internationales en faveur des mobilités étudiantes.

5 Sans ignorer ces facteurs, nous nous attacherons ici à montrer, comme l'ont fait d'autres auteurs pour des pays comme le Pérou (Daniel, 2014), que des dynamiques collectives, enclenchées par les étudiants et la communauté scientifique, président aussi à ces mobilités. Nous verrons que, de génération en génération, les chercheurs mexicains ont en effet tissé des liens privilégiés avec certaines institutions étrangères de formation, y envoyant leurs étudiants à leur suite par adhésion aux enseignements dispensés, aux écoles de pensée et à leurs représentants universitaires. Les mobilités à l'étranger des étudiants mexicains sont, en quelque sorte, un héritage de leurs aînés formés à l'étranger et le résultat d'une attraction des "pôles de savoir " (Gérard et Maldonado, 2009) que représentent les pôles de formation en raison des enseignements dispensés en leur sein.

6 L'extension du marché international de la formation et l'intensification des politiques nationales d'internationalisation suggèrent toutefois la transformation des dynamiques et logiques de mobilités étudiantes. Celles-ci se déploient en effet de plus en plus au sein de cadres de coopération, de sorte qu'elles paraissent davantage officiellement "encadrées" que par le passé, et peut-être moins intégrées aujourd'hui à des dynamiques scientifiques impulsées par les chercheurs et universitaires eux-mêmes indépendamment des dispositifs institutionnels de coopération. Nous nous interrogerons en dernier lieu sur une telle évolution des mobilités pour formation.

$7 \quad$ Le cas des ingénieurs mexicains membres du Système national mexicain des chercheurs (Sistema Nacional de Investigadores - SNI) sera analysé dans cette étude. Créé en 1984 dans la perspective d'éviter la "fuite des cerveaux ", de consolider le corps des enseignantschercheurs, enfin de parfaire la formation au plus haut niveau des enseignantschercheurs mexicains, ce système comptait près de 16600 membres en 2010, dont 2249 ingénieurs (Didou et Gérard, 2010). Constituée grâce aux CV des enseignants chercheurs, la base du SNI permet de connaître leurs caractéristiques socio- 
démographiques de base (sexe, âge) et plusieurs critères relatifs à leur parcours de formation, en particulier le lieu géographique, l'institution, la date et la discipline d'obtention des diplômes d'enseignement supérieur ${ }^{1}$.

Deux séries de données statistiques appuieront cette exploration : en premier lieu, des données sur la composition du "corps" des ingénieurs, appréciée à travers le siècle passé jusqu'à aujourd'hui. En deuxième lieu, des indicateurs grâce auxquels peuvent être identifiés les pôles de formation des chercheurs : les lieux de formation, la date et la génération d'obtention des diplômes, la discipline des chercheurs, enfin le lieu de leur exercice professionnel actuel.

Des entretiens ${ }^{2}$ nous permettront d'affiner l'analyse des principaux facteurs qui contribuent à la décision d'aller étudier à l'étranger et qui déterminent le "choix" de la destination.

10 Après avoir rapidement identifié le champ de l'ingénierie mexicaine, nous nous focaliserons sur les mobilités à l'étranger des élèves-ingénieurs, puis nous nous interrogerons plus fondamentalement sur les facteurs et dynamiques de ces mobilités. Ces développements nous conduiront à interroger les transformations des mobilités et le passage d'une logique d'“héritage", par les étudiants, des choix opérés en matière de formation par leurs aînés, à une logique distincte caractérisée par un "encadrement" plus officiel des mobilités.

\section{L'ingénierie mexicaine : une "science" en développement depuis plusieurs siècles}

11 Au Mexique, l'enseignement de l'ingénierie date de 1772 avec le "Séminaire royal des mines" (Real Seminario de Minas), alors le premier institut de recherche scientifique du continent, institué pour répondre au problème de la baisse de production d'argent occasionnée par le manque de techniques d'extraction minière. La couronne d'Espagne y était particulièrement attentive, dans la mesure où le commerce et les métaux précieux étaient les premières sources de son patrimoine (Aldama, 2009). Les caractéristiques climatologiques, géologiques et sismiques du Mexique, jointes à des données historiques, ont par ailleurs sous-tendu, depuis la colonisation (16é-17è siècles), une large tradition de développement de certaines disciplines d'ingénierie et leurs hauts nivaux de reconnaissance internationale.

L'ancien collège des Mines a développé les domaines de l'ingénierie métallurgique, de l'ingénierie civile et de l'hydraulique, en parallèle à la formation d'autres professions nécessaires pour la gestion de la nouvelle Espagne, comme la comptabilité, le droit et la médecine. Ces professions ont, de longue date, été prestigieuses, y compris aux yeux des populations faiblement scolarisées et sont, aujourd'hui encore, considérées comme de réelles voies de mobilité sociale ${ }^{3}$. Une des spécialités d'ingénierie civile dans laquelle le Mexique occupe une place prépondérante est celle des infrastructures. Le pays représente, aux yeux de nombreux experts, un laboratoire vivant en raison des mouvements des plaques tectoniques et autres effets, sur le niveau des sols, des déplacements, fragilisations ou effondrements des constructions. Les disciplines d'ingénierie nées d'applications des sciences fondamentales ont été, elles, introduites plus tardivement dans le pays et, bien que certaines d'entre elles, comme les sciences 
du calcul (computación), sont fortement demandées par les étudiants, la recherche dans ces domaines est moins développée et est l'objet d'une moindre reconnaissance.

$\mathrm{Au}$ milieu du vingtième siècle, la nécessité de moderniser les infrastructures et les services puis, un peu plus tard, la découverte de grands gisements pétrolifères, ont enclenché le développement du champ de l'ingénierie, qui n'a cessé de se diversifier. À la fin des années 1990, celui-ci était ainsi le second domaine disciplinaire (áreas), sur les 7 que distingue le CONACYT au niveau de la licence, avec 32,5\% (481 000 étudiants) des effectifs totaux inscrits à ce niveau (ANUIES, 2003). La Zone Metropolitaine de la ville de México (ZMCM) comptait alors 85 licences en relation avec la technologie et l'industrie (ibid). Les structures d'enseignement de l'ingénierie se sont également multipliées et diversifiées : le système d'enseignement technique pour la formation dans ce domaine, en deçà et au niveau de la licence, comprend désormais les universités fédérales, les universités d'Etat, les instituts technologiques fédéraux et d'Etat, les universités technologiques, les universités polytechniques, ou encore les universités et instituts privés (principalement l'Institut Technologique d'Etudes Supérieures de Monterrey ITESM) ${ }^{4}$. Notons enfin que, entre 2008 et 2012 , respectivement $32,7 \%$ et $28,8 \%$ des bourses accordées pour les études de maestria et de doctorat par le CONACYT mexicain ont été destinées aux étudiants en ingénierie.

Le champ de l'ingénierie a par ailleurs été récemment sujet à l'émergence de nouveaux domaines de recherche et de technologie : ingénierie des matériaux - dans laquelle s'intègrent des éléments de physique et de chimie -, biotechnologie - dans laquelle la génétique, la biologie, la zootechnologie et l'ingénierie jouent un rôle fondamental -, nanotechnologie (biologie ou chimie moléculaire) ou encore ingénierie en systèmes intelligents (robotique, intelligence artificielle, etc.).

Ces développements indiquent la complexification croissante de ce champ. Ils peuvent de surcroît être interprétés comme le produit d'une circulation des savoirs - des pays où se forment les chercheurs mexicains vers le Mexique - et, à la fois, comme la capacité accrue du Mexique à développer de tels domaines scientifiques, en partie grâce à l'apport des scientifiques formés à l'étranger - donc grâce aux mobilités pour formation au fil des générations.

\section{La mobilité à l'international pour études : une donnée structurelle de l'ingénierie}

16 La formation à l'étranger des chercheurs mexicains est une composante historique dans la structuration de la recherche. À l'échelle du siècle passé, près de $40 \%$ des chercheurs membres du SNI ont obtenu leur doctorat à l'étranger (Didou et Gérard, op. cit.). Les chercheurs-ingénieurs ${ }^{5}$ ne font pas exception : leur formation au plus haut niveau est, toutes générations confondues, presque autant "exogène" qu'"endogène". $44,7 \%$ d'entre eux a en effet obtenu son doctorat à l'extérieur du Mexique. L'ingénierie est ainsi le domaine disciplinaire dont la proportion de chercheurs formés à l'étranger est la plus importante ${ }^{6}$.

17 Peu à peu, le Mexique a acquis des capacités de formation au plus haut niveau et a pris le pas sur les pays étrangers pour la formation de ses docteurs, quelle que soit leur discipline. Même s'ils ont été en butte aux crises économiques (1982), les plans de développement institutionnel de l'enseignement supérieur et de la recherche se sont en 
effet imposés avec force. Entre 1960 et 2010 par exemple, le nombre d'établissements d'enseignement supérieur a crû de 78 à 3745 , les étudiants inscrits en licence sont passés de 78000 à 2599 253, le taux brut de couverture (tbc) de l'éducation supérieure ${ }^{7}$ passant de 2,7 \% à 27,1 \% durant cette même période (Grediaga et Maldonado, 2012).

Cependant, le mouvement a été progressif et tardif. En raison du mode de développement du système d'éducation supérieure sur le territoire national, les études étaient notamment limitées en dehors des grands centres urbains (Grediaga, 2012). Le développement du posgrado (au-delà de la licence) dans le pays s'accompagnait notamment, pour les natifs de villes éloignées de la capitale ou des principales villes du pays, d'une méconnaissance totale des possibilités, voire du sens même d'entreprendre des études de posgrado. Un ingénieur de la UAM à Mexico, d'une soixantaine d'années, de niveau SNI 3, l'exprime en ces termes :

«Nous ne savions pas vraiment ce qu'étaient des études de posgrado, parce qu'aucun de nos professeurs n'en avait fait. J'étais alors dans une institution où il n'y avait pas de bourses; il fallait aller les chercher à l'extérieur. Ma famille était enseignante, elle pouvait me soutenir mais peu, de sorte que je suis allé me renseigner à l'école de posgrado de la UNAM. Celui qui était alors le secrétaire - qui venait de rentrer du MIT -, m'a accordé un entretien et m'a dit qu'il allait voir comment la fondation des ingénieurs accordait des bourses. Bien que cela n'était pas encore assuré pour moi, je me suis inscrit en maestria ".

Cette absence de structures nationales de formation de posgrado enclenche très tôt la mobilité pour formation à l'étranger, la majorité des ingénieurs se formant alors en dehors du Mexique durant plusieurs décennies, jusqu'aux années 1990 (Graphique 1). Dans le domaine de l'ingénierie, cette évolution globale mérite toutefois d'être nuancée en considération d'inflexions distinctes selon ses disciplines. Les étudiants de certaines d'entre elles se forment majoritairement au Mexique dès les années 1980 (informatique); dans d'autres au contraire (ingénierie des matériaux), ils se forment durablement à l'étranger. Il faut attendre le début des années 1990, voire cinq ans plus tard (sciences du calcul, ingénierie de l'environnement) pour que le Mexique prenne le relais des pays étrangers dans la formation de ses ingénieurs.

Graphique 1 : Répartition des ingénieurs du SNI par principaux pays de formation et par décennie d'obtention du doctorat, Mexique inclus

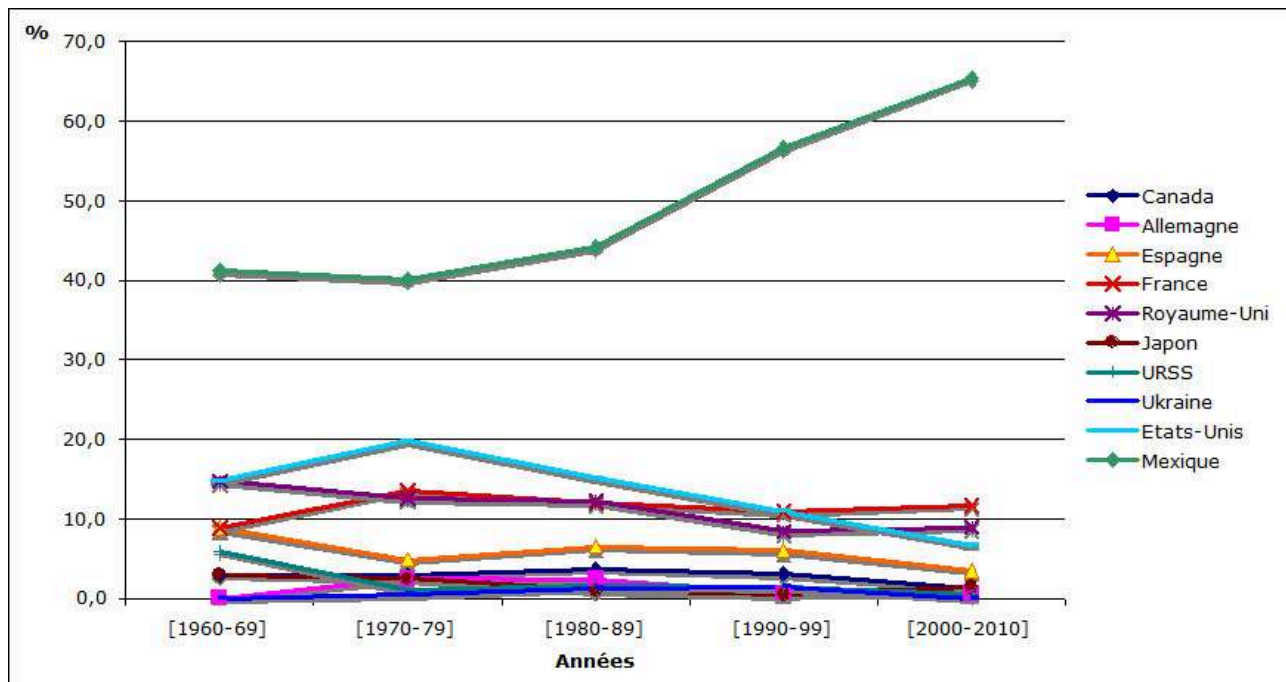

Source : d'après la base SNI, 2009. 
Ces évolutions contrastées signalent des configurations disciplinaires différenciées dans le temps et dans l'espace. De décennie en décennie, changent les pays étrangers de formation des étudiants ingénieurs. Comme pour l'ensemble des chercheurs mexicains, quatre d'entre eux font figure de principaux pays d'accueil des élèves ingénieurs : les Etats-Unis, la France, le Royaume-Uni puis, plus tard, l'Espagne. Au fil des générations cependant, les mobilités de ces étudiants se sont réorientées (graphique 2). Certains pays, comme l'URSS et d'autres pays d'Europe de l'Est, ont attiré de nombreux élèves ingénieurs jusqu'aux années 1990, avant de perdre leur position d'importants pôles de formation. Parallèlement, certains pays ont peu à peu conquis une place prépondérante, comme la France.

Graphique 2 - Répartition des ingénieurs du SNI par principaux pays étrangers de formation et par décennie d'obtention du doctorat

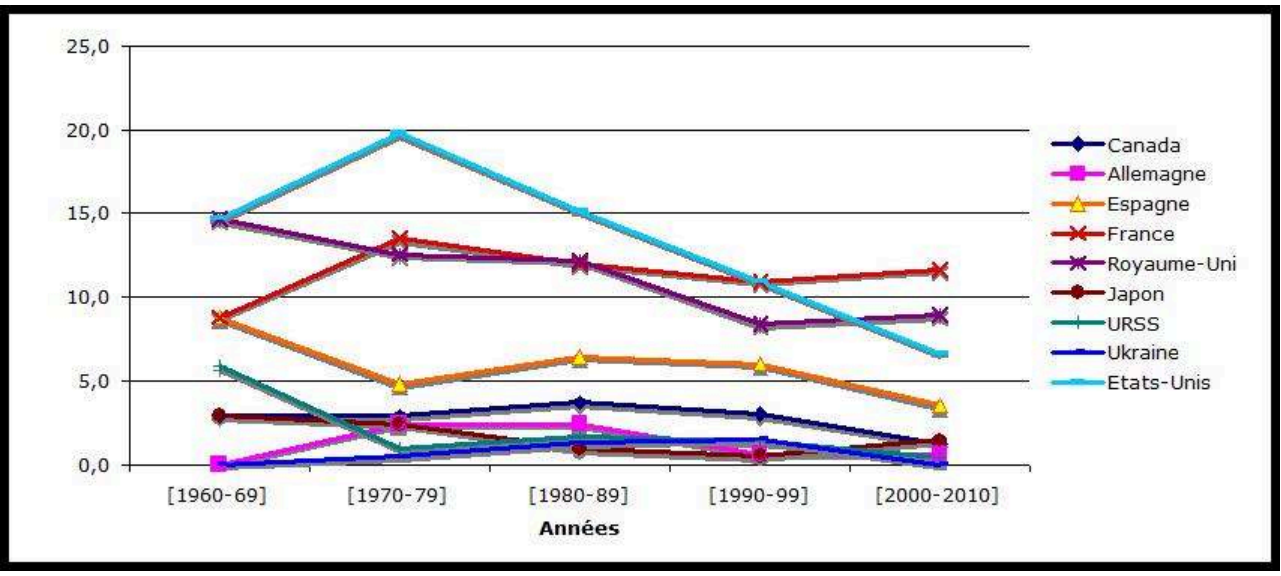

SOURCE : D'APRÈS LA BASE SNI, 2009.

21 Les hiérarchies des pôles de formation se sont aussi transformées au fil des décennies. De pôle dominant, les Etats-Unis sont devenus dans certaines disciplines un pôle secondaire, comme en chimie, en électronique, en sciences du calcul. La perte de leur suprématie dans la formation des élèves-ingénieurs s'est accompagnée d'une mobilité étudiante plus forte vers l'Europe, en particulier vers la France et le Royaume-Uni. Au niveau de l'ensemble des chercheurs SNI et dans la décennie 2000, l'Espagne a devancé la France pour former des chercheurs mexicains. Concernant les ingénieurs et pour cette même décennie, la France constituait le principal pôle de formation (graphique 2).

Des distinctions plus fines par disciplines méritent d'être réalisées (graphique 3). Si la France est le principal pays étranger de formation pour l'ingénierie de l'alimentation, de l'informatique, de l'environnement, ou encore de la chimie, le Royaume-Uni et les Etats-Unis constituent les principales destinations des étudiants qui suivent une formation en ingénierie des matériaux. Ceux qui entreprennent un doctorat d'ingénierie électronique à l'étranger se rendent en proportions égales en France et aux Etats-Unis, mais à peine plus qu'en Espagne ou au Royaume-Uni. De même, pour les sciences du calcul (computación), la France, le Royaume-Uni et les Etats-Unis constituent des pôles de formation d'égale importance.

Pour certaines disciplines, les pôles étrangers de formation des élèves ingénieurs sont ainsi hiérarchisés et constants (ingénierie de la chimie ou des matériaux par exemple). 
Pour d'autres en revanche, aucun pôle de formation ne constitue une destination privilégiée et pérenne d'une génération à l'autre des élèves ingénieurs. Pour ces formations, les destinations étrangères sont davantage aléatoires et les flux d'élèves ingénieurs paraissent obéir à des facteurs sujets à variation, comme le coût respectif des études dans les différents pays. Ces disciplines sont multipolaires, à l'inverse de celles qui se distinguent par un pôle étranger de formation dominant dans l'espace international de la formation.

Graphique 3 - Répartition des ingénieurs du SNI (toutes générations) par principaux pays de formation $(+3 \%)$ et pour les principales disciplines d'ingénierie $(+5 \%)$

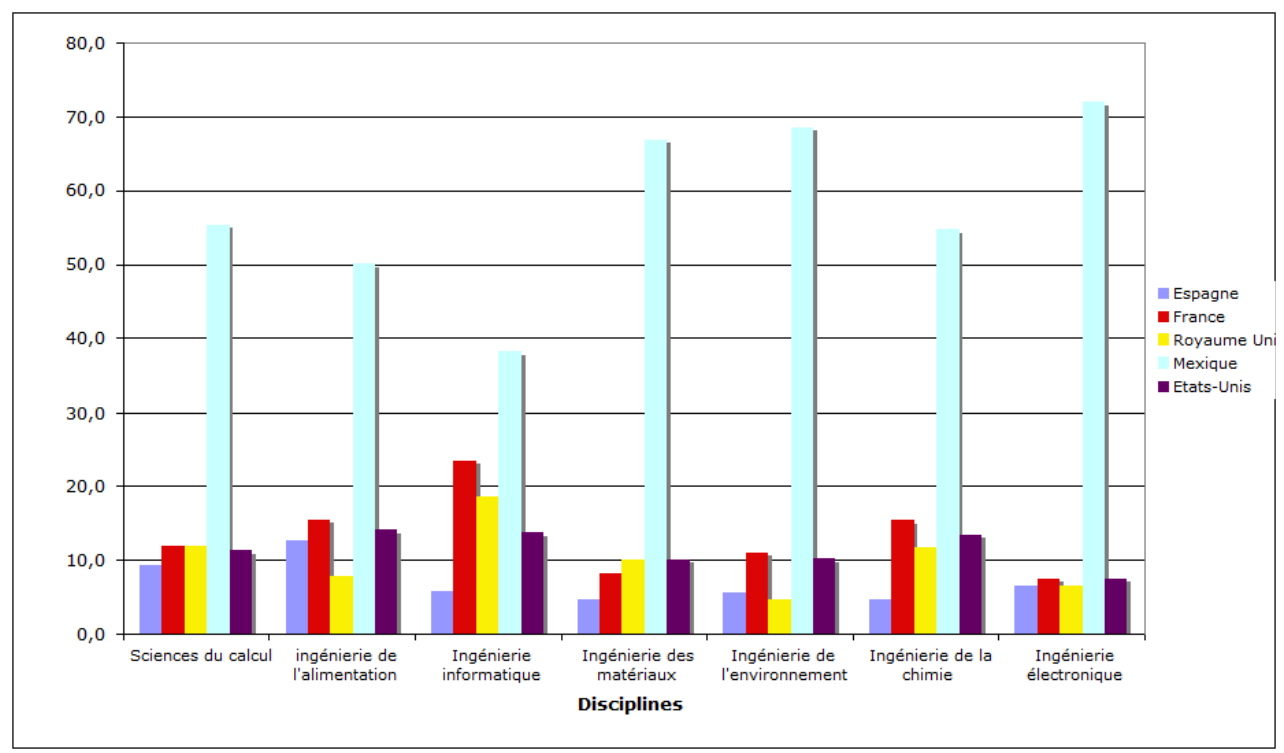

SOURCE : D'APRÈS LA BASE SNI, 2009.

NB1. Ne sont retenus ici que les pays étrangers où plus de 3 \% d'ingénieurs mexicains du SNI se sont formés en raison de la très faible représentation des autres pays étrangers de formation (voir annexe 1).

NB2. Seules sont prises en compte les disciplines d'ingénierie qui comptent plus de 5\% d'ingénieurs faute de représentativité des autres disciplines.

Certains pôles étrangers de formation sont ainsi particulièrement attractifs. La spécialisation de ces pôles les a transformés en pôles de référence (Gérard et Cornu, 2013 ; Razafimahefa et Raynal, 2014). Au regard des ingénieurs SNI formés dans cette discipline, l'ingénierie de l'informatique paraît, dans une certaine mesure, marquée par l'influence française; l'ingénierie des communications, par l'influence britannique, l'ingénierie de l'urbanisme, par l'influence espagnole. Cette attraction s'explique en partie par le degré de spécialisation des pays dans certains domaines (graphique 4). L'informatique apparaît par exemple comme le principal domaine suivi par les ingénieurs SNI formés en France; l'ingénierie de l'alimentation comme le principal domaine de formation en Espagne, etc. 
Graphique 4 - Répartition des ingénieurs du SNI (toutes générations) par disciplines d'ingénierie (+ $5 \%$ ) dans les principaux pays de formation $(+3 \%)$

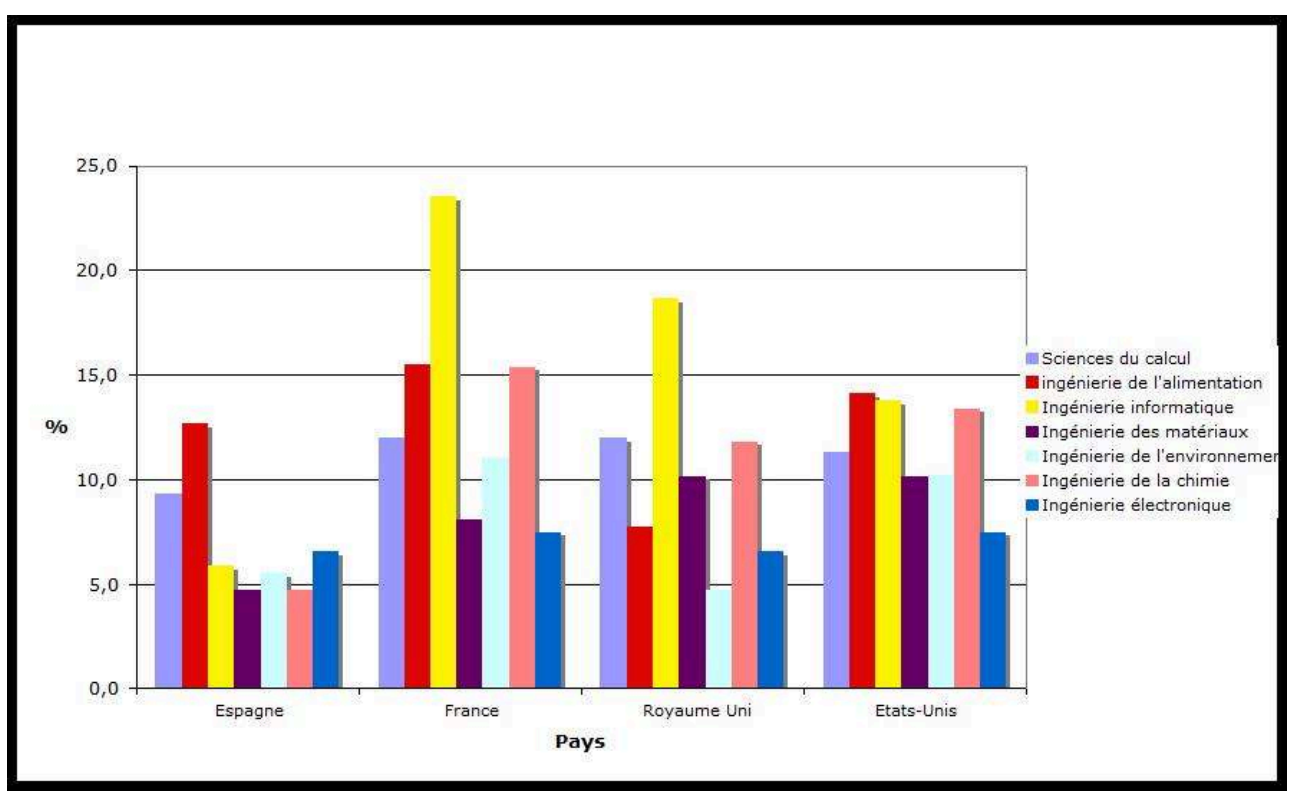

Source : d'après la base SNI, 2009.

NB1. Ne sont retenus ici que les pays étrangers où plus de $3 \%$ d'ingénieurs mexicains du SNI se sont formés en raison de la très faible représentation des autres pays étrangers de formation (voir annexe 1).

NB2. Seules sont prises en compte les disciplines d'ingénierie qui comptent plus de 5\% d'ingénieurs faute de représentativité des autres disciplines.

Les institutions cherchant à développer des programmes de posgrado et les pionniers ayant couronné leurs études à l'étranger ont à cet égard joué un rôle fondamental, les premières bénéficiant du capital des seconds et de leur capacité à développer des programmes d'enseignement et des groupes de recherche dans le pays. Pour l'analyse, les lieux d'études - tant à l'étranger qu'au Mexique - ont ainsi toute leur importance.

\section{Pôles de formation et "chaînes de savoir"}

L'examen du nombre de doctorats délivrés par les Etats-Unis, la France, l'Angleterre et l'Espagne, comptabilisés pour les ingénieurs du SNI (annexe 1), révèle deux tendances dominantes caractéristiques du champ de la formation en ingénierie. Il s'agit, d'une part, de l'“endogénéisation" croissante de la formation au Mexique et, d'autre part, de l'existence de liens pérennes entre le Mexique et certains pays étrangers de formation, qui témoignent d'une institutionnalisation progressive des relations de coopération. En Espagne, par exemple, les universidades politecnicas de Catalunya, Madrid et Valencia ont formé $51,3 \%$ des étudiants mexicains en ingénierie qui se sont dirigés vers ce pays et qui sont actuellement membres du Sistema Nacional de los Investigadores. En France, le réseau Polytech, à travers ses écoles de Grenoble, Toulouse et Nancy a diplômé 39,5 \% de ceux qui, membres de dernier système, se sont formés dans ce pays. L'université de Manchester a formé plus de $20 \%$ des élèves ingénieurs mexicains du SNI qui se sont rendus au Royaume-Uni. Dans certains Etats du Mexique, l'étude des lieux de formation des ingénieurs met au jour des liens privilégiés des principales institutions mexicaines de formation d'ingénieurs avec des homologues étrangères. Tel est par exemple le cas 
du Veracruz, où la majorité des ingénieurs chimistes se sont formés en France et, parmi eux, une majorité à l'Institut Polytech de Toulouse.

Tel est aussi le cas d'institutions de formation de Mexico, comme le CINVESTAV (Centre de recherches et d'études avancées de l'Institut polytechnique national) avec des écoles étrangères, comme l'institut Polytech de Grenoble. Le CINVESTAV a formé, au niveau du master, $47,1 \%$ des ingénieurs du SNI. Parmi ces derniers, $41 \%$ ont réalisé un doctorat à l'étranger, et $42 \%$ d'entre eux se sont dirigés vers la France. Près de $20 \%$ de ces derniers sont allés étudier à l'institut Polytech de Grenoble, l'institution qui, à l'échelle des ingénieurs du SNI, a accueilli la proportion la plus importante de ceux qui se sont rendu en France pour y entreprendre un doctorat. Cette "chaîne » entre le CINVESTAV mexicain et l'école Polytech de Grenoble a aujourd'hui quarante ans: le premier des lauréats de l'école a été diplômé en 1971.

Des exemples fournis par des enseignants et chercheurs mexicains d'autres disciplines attestent d'une telle logique de reproduction, par les étudiants, de mobilités vers des pôles précis de formation. Sensibilisés par leurs enseignants formés à l'étranger à des écoles de pensée particulières, aux théories de chercheurs étrangers convertis en de véritables " figures tutélaires ", mais aussi démunis, durant des décennies, par la rareté des cadres officiels de coopération et par la méconnaissance des opportunités réelles d'étudier dans les différents pays, de nombreux étudiants ont ainsi emprunté les circuits suivis par leurs aînés et ont légué à leurs élèves leur connaissance du milieu étranger où ils se sont formés. Un ingénieur de l'université Unison raconte :

«Une professeure (maestra) qui était à l'université de Massaschussets est venue ici,

à l'université ; résultat : je suis allé faire un séjour dans son université, je me suis entretenu avec le docteur X, qui m’a alors invité à y faire le doctorat. Il me dit : “Tu sais, le projet que tu amènes est vraiment bien, cela m'intéresse. Si ça te dit, je t'obtiens une bourse ici". Bon, personne ne réalisait alors de doctorat, et encore moins à l'étranger, encore moins dans une autre langue, mais, en 1994, sans savoir parler anglais, ou très peu, je suis allé là-bas ».

Un autre ingénieur de la UNAM, d'une trentaine d'années, souligne comment il est allé étudier en France :

«J'ai été attiré par une amie pour faire mon service social à l'institut d'ingénierie. Elle y était enseignante et travaillait avec une chercheure titulaire d'un doctorat qu'elle avait fait en France (...). Je crois que celle-ci vit quelque chose en moi, elle me dit: "Pourquoi tu ne vas pas faire tes études en France"? Je lui dis: "Mais je n'ai jamais étudié le français !". Et elle me dit: "Ecoute, il y a une proposition [convocatoria] conjointe France-Mexique du Conacyt, et ceux qui sont sélectionnés suivent un cours intensif de français". J'ai trouvé tout ça très bien et je me suis décidé [à aller faire le doctorat en France] ».

Tant les professeurs que les étudiants résidant à l'étranger tracent ainsi la voie vers des pôles particuliers de formation, qu'empruntent à leur tour leurs élèves et camarades. Comme le soulignent Mazzella et Eyebiyi (2014: 24), «la place et le rôle de ces prescripteurs dans la chaîne migratoire se trouvent [ainsi] éclairés d'un jour nouveau, faisant d'eux non seulement les porte-voix nécessaires des institutions officielles, mais aussi et surtout une sorte de caution informationnelle déterminante dans l'attractivité de ces nouvelles destinations ".

31 Ces pôles de formation tirent leur attraction, dans le cas des sciences humaines au moins, des savoirs incarnés par des écoles de pensée particulière et leurs représentants. L'économie en fournit un exemple : de son courant marxiste, enseigné notamment en 
France dans les années 1970 et 1980 et largement repris par l'institut d'économie de la UNAM, à celui d'inspiration néo-libérale anglo-saxonne, enseigné au CIDE notamment, elle illustre cette relation entre pôles de formation, mobilité des étudiants et structuration du champ scientifique mexicain. Elle révèle en ce sens le poids déterminant des écoles et institutions étrangères en tant que " pôles de savoirs » dans la configuration des mobilités étudiantes et, au-delà, l'origine des liens tissés entre institutions étrangères et mexicaines de formation et de recherche; des liens que l'on peut désigner comme des " chaînes de savoir " (Gérard et Maldonado, 2009), peu à peu et informellement tissées par les chercheurs et étudiants mobiles - avant d'être parfois formalisées par des accords de coopération. Vecteurs de circulation d'écoles de pensée, mais aussi de pratiques scientifiques, ces « chaînes » ont participé à la structuration du champ scientifique et à l'émergence, en son sein, de «niches disciplinaires » marquées par des théories particulières.

De telles « chaînes » ont également été structurantes dans le domaine de l'ingénierie. Par exemple, les chimistes travaillant dans une même institution mexicaine et qui se sont formés en France ont, dans la majorité des cas, obtenu leur doctorat dans la même institution. Ainsi, les deux ingénieurs de la direction générale de l'éducation supérieure technologique de Basse Californie, formés en France, sont issus de l'institut polytechnique de Toulouse; les deux lauréats de diplômes français qui travaillent à l'université autonome métropolitaine se sont également formés à l'université de Lyon 1 , ceux de l'institut polytechnique national se sont diplômés à l'université de Montpellier 2, etc. Apparaissent ce que l'on peut désigner comme des « communautés de formation » intergénérationnelles, qui donnent lieu, au Mexique, à des groupes ou " communautés professionnelles " partageant une affiliation commune à l'institution étrangère dans laquelle ils se sont formés.

Les « chaînes » tissées entre les différents pays étrangers et le Mexique sont cependant variables et inégalement structurelles. Deux données en témoignent. D'une part, les élèves-ingénieurs qui se forment en France et en Espagne semblent inscrits dans des chaînes de mobilité et de savoir consolidées de longue date, davantage que les ingénieurs mexicains formés dans les pays anglophones (Etats-Unis et Royaume-Uni). Ces derniers retournent en effet davantage au Mexique après avoir réalisé un master à l'étranger ( $26 \%$ d'entre eux, contre $8 \%$ et $5 \%$ pour l'Espagne et la France). D'autre part, le diplôme et le type de formation étrangers priment en certains cas sur l'institution étrangère de formation au moment de l'insertion professionnelle au Mexique. Cela s'observe par exemple dans le cas des ingénieurs du Nuevo Léon: majoritairement formés à l'ITESM au niveau de la licence, ces derniers poursuivent aussi en majorité leur formation aux Etats-Unis; mais, à la différence des ingénieurs du Veracruz formés en France, ils ne se forment pas dans une institution particulière avec laquelle l'ITESM aurait des liens privilégiés. Ce dernier institut, qui recrute majoritairement les ingénieurs formés en son sein jusqu'à la licence, privilégie ainsi le type de formation - en l'occurrence, américaine - à la source (institution) de la formation.

Ces exemples suggèrent des liens différenciés entre le Mexique et les pays francophones et hispanophones, d'une part, et les pays anglophones, d'autre part; ils témoignent aussi des logiques complexes de mobilité des étudiants: en partie dynamisées par des "chaînes de savoirs " historiquement constituées, ces dernières obéissent aussi à la légitimité symbolique des diplômes délivrés par les différents pays 
et, à un autre niveau, aux politiques conjoncturelles de coopération entre le Mexique et les différents pays étrangers.

\section{« Chaînes de savoir » et reconfiguration des mobilités pour formation à l'international}

Les entretiens réalisés auprès d'ingénieurs mexicains montrent l'importance actuelle du marché international de la formation et des logiques d'attractivité sur la configuration des mobilités pour formation. S'il était auparavant "naturel" pour les professeurs de recommander aux meilleurs étudiants d'aller se former là où euxmêmes avaient étudié, ils examinent aujourd'hui davantage, avec les candidats au doctorat, les alternatives possibles au Mexique ou à l'étranger. Outre l'expérience acquise par les ingénieurs mexicains dans l'espace scientifique international, les nouveaux critères d'octroi des bourses par le CONACYT (sous l'influence du PNPC notamment ${ }^{8}$ ), ou encore le développement de rankings par champ de recherche contribuent à changer les représentations des uns et des autres des opportunités, conditions et possibilités de la mobilité - ce pour quoi n'opère plus toujours le processus de reproduction, par les étudiants mobiles, des voies ouvertes par leurs prédécesseurs. Et la prolongation des relations entre chercheurs mexicains et étrangers dépend à la fois « de la complicité intellectuelle et de l'adhésion aux mêmes courants scientifiques, et aux coopérations bilatérales » (Agulhon, 2015 : 92).

Dans certains cas interviennent ainsi les opportunités offertes par les conventions ou accords entre pays ou institutions ${ }^{9}$. Dans d'autres cas, les mobilités à l'international répondent à des données conjoncturelles et à des logiques sociales de mobilité, en particulier familiales : le «capital cosmopolite» des individus sous-tend en effet leurs mobilités à l'international (Wagner, 1998). À ce titre, les mobilités ne peuvent pas être analysées seulement en référence aux politiques d'internationalisation de l'enseignement supérieur. Doivent également être étudiés leurs contextes et facteurs sociaux de production.

Cette pluralité de facteurs et motivations à l'origine des études à l'étranger s'intègre au cadre général du marché international et des espaces nationaux de formation. À mesure que le premier s'est ouvert et diversifié, les mobilités à l'international se sont multipliées; conjointement, les affiliations anciennes à des centres étrangers de formation se sont transformées et semblent être peu à peu accompagnées de logiques distinctes de mobilité: les accords bilatéraux de coopération entre institutions mexicaines et étrangères de formation se multiplient en effet, et la part des mobilités étudiantes "spontanées" diminue au regard des mobilités encadrées par les dispositifs officiels de coopération (Agulhon, 2015). Et ce processus de transformation n'est pas achevé si l'on considère la croissance des bourses accordées par le Conacyt et les institutions étrangères pour des études à l'étranger ${ }^{10}$, la mise en place de dispositifs nouveaux comme les bourses mixtes, ou encore la multiplication des séjours courts (sous forme de stage) mis en place par les institutions de formation elles-mêmes, mexicaines et étrangères, au titre de leur coopération. 


\section{Conclusion}

Les politiques mexicaines d'internationalisation de l'enseignement supérieur participent depuis des décennies au développement des mobilités étudiantes et, consécutivement, au processus de consolidation du champ scientifique. Les données mobilisées et analysées ici mettent au jour deux autres ressorts essentiels de ces mobilités: d'une part, la structuration du champ de la recherche par des pays étrangers, d'autre part la capacité des "communautés " académiques et scientifiques, mexicaines et étrangères, à tisser des liens pérennes entre elles. Ainsi, les cadres politiques et institutionnels ne peuvent pas être retenus comme seuls facteurs de la configuration des mobilités à l'international pour formation. L'historicité des liens entre milieux académiques mexicains et étrangers, les réseaux internationaux et les conditions de transmission du savoir à l'étranger sont tout aussi importants.

À l'analyse, ces réseaux et "chaînes de savoir » tissées entre les " communautés » et institutions académiques mexicaines et étrangères ont été particulièrement structurants de ce champ scientifique. Mais leur configuration s'est transformée : les mobilités pour formation sont aujourd'hui davantage institutionnellement «encadrées" et les dynamiques scientifiques «segmentées». En dépit de cette évolution, des réseaux internationaux se tissent, de nouvelles lignes de recherche sont initiées par les lauréats de diplômes étrangers. La science se transforme et, faute de toujours pouvoir s'exporter ou se développer avec des communautés scientifiques étrangères - en raison d'inégales conditions de production scientifique notamment -, elle s'internationalise de l'intérieur, en quelque sorte. Dans le cadre des travaux consacrés à l'impact des mobilités étudiantes sur la structuration du champ scientifique national et la définition des objets, lignes et méthodes de recherche dans des domaines comme l'ingénierie, cette tension entre l'internationalisation de l'enseignement supérieur et les dynamiques endogènes de développement scientifique mérite toute l'attention.

\section{BIBLIOGRAPHIE}

Agulhon, Catherine, "circulacion de los indindividuos-circulacion de los saberes Francia-México, de una generacion a la otra", in Sylvie Didou et Pascal Renaud (dir), Circulacion internacional de los conocimientos: miradas cruzadas sobre la dinamica Norte-Sur, Unesco-Iesalc, 2015, p. 71- 94.

Aldama, Ávalos Daniel, Vinculación de la educación superior con el sistema productivo nacional: El caso de los ingenieros mecánicos de la FES-Aragón, México, UNAM, tesis de doctorado, 2009.

ANUIES (Asociación Nacional de Universidades e Instituciones de Educación Superior), Anuario estadístico. Población escolar y personal docente en la educación media superior y superior, ciclo escolar 2010-2011, México, 2012.

ANUIES (Asociación Nacional de Universidades e Instituciones de Educación Superior) (2003), Anuarios Estadísticos de la Educación Superior. Licenciatura, México, 2003. 
Charle, Christophe, Schriewer Jurgen et Wagner Peter, dirs, Redes intelectuales trasnacionales. Formas de conocimiento académico y búsqueda de identidades culturales, Barcelona, Pomares, 2006.

Daniel, Camila, « Building a South-South connection through Higher Education: the case of Peruvian university students in Brazil », Cahiers de la Recherche sur l'éducation et les savoirs, $\mathrm{n}^{\circ} 13$, 2014, p. 119-137.

Didou Aupetit, Sylvie et Gerard, Etienne, El sistema nacional de investigadores, veinticinco años después. La comunidad científica, entre distinción e internacionalización, México, ANUIES, 2010.

Foro Consultivo Científico y Tecnológico (FCCYT), Análisis de los parámetros de evaluación del Programa Nacional de Posgrados de Calidad por área del conocimiento. Grupo de Análisis del Posgrado y Subgrupos de Análisis del Posgrado por Área del conocimiento. Reporte entregado a CONACYT, Junio, Mexico, D.F., 2009.

Gérard, Etienne et Cornu, Jean-François, « Dynamiques de mobilité étudiante Sud-Nord : une approche par les pôles internationaux de formation de l'"élite" scientifique mexicaine ", Cahiers québecois de démographie, vol. 42, n², 2013, p. 241-272.

Gérard, Etienne et Maldonado, Estela, « "Polos de saber” y “cadenas de saber”. Un análisis del impacto del proceso de movilidad académica entre México y el extranjero », Revista de la Educación Superior, ANUIES, n52, 2009, p. 49-64.

Grediaga, Rocío, « La configuración del sistema de educación superior en México: políticas públicas y desarrollo institucional », in Carlos Pallán et Roberto Rodríguez (Coords.), La SEP en el desarrollo de la educación superior, México, FCE, 2012, p. 1-58.

Grediaga, Rocío et Maldonado, Estela, « Une première approche de la reconstruction des principaux pôles internationaux de formation des scientifiques mexicains à partir de 1960 ", Cahiers de la Recherche sur l'éducation et les savoirs, n¹1, 2012, p. 73-106.

Mazzella, Sylvie \& Eyebiyi, Elieth P., « Observer les mobilités étudiantes Sud-Sud dans l'internationalisation de l'enseignement supérieur ", Cahiers de la Recherche sur l'éducation et les savoirs, $\mathrm{n}^{\circ} 13,2014$, p. 7-24.

Moulinier, Pierre, Les étudiants étrangers à Paris au XIXè siècle. Migration et formation des élites, Rennes, Presses Universitaires de Rennes, 2012.

Razafimahefa, Lala et Raynal, Jean-Claude, « Dynamiques des réseaux de mobilité étudiante entre pays du Sud : attractivité, compétitivité et multipolarité », Cahiers de la Recherche sur l'éducation et les savoirs, $\mathrm{n}^{\circ} 13,2014$, p. 25-52.

Vinokur, Annie et Sigman, Carole (dirs), « L’enseignement supérieur entre nouvelle gestion publique et crise systémique », Economie et Sociétés, hors-série ${ }^{\circ} 43, n^{\circ} 4,2010$.

Wagner (A.-C.), Les nouvelles élites de la mondialisation, PUF, 1998.

\section{ANNEXES}

Annexe 1 : Répartition des chercheurs-ingénieurs du SNI (en 2009) par pays d'obtention du doctorat

\begin{tabular}{|l|l|l|l|}
\hline & $\mathrm{N}$ & $\%$ & $\%$ cumulé \\
\hline
\end{tabular}




\begin{tabular}{|c|c|c|c|}
\hline Mexique & 1144 & 50,867 & 50,867 \\
\hline Etats-Unis & 257 & 11,427 & 62,294 \\
\hline France & 245 & 10,894 & 73,188 \\
\hline Royaume-Uni & 214 & 9,515 & 82,703 \\
\hline Espagne & 114 & 5,069 & 87,772 \\
\hline Canada & 60 & 2,668 & 90,44 \\
\hline Allemagne & 28 & 1,245 & 91,685 \\
\hline Russie & 27 & 1,201 & 92,886 \\
\hline Japon & 23 & 1,023 & 94,042 \\
\hline Ukraine & 20 & 0,889 & 94,931 \\
\hline Pologgne & 10 & 0,445 & 95,376 \\
\hline NLD & 7 & 0,311 & 95,687 \\
\hline Chine & 6 & 0,267 & 95,954 \\
\hline Belgique & 5 & 0,222 & 96,176 \\
\hline Brésil & 5 & 0,222 & 96,398 \\
\hline Cuba & 5 & 0,222 & 96,621 \\
\hline IND & 5 & 0,222 & 96,843 \\
\hline Italie & 5 & 0,222 & 97,065 \\
\hline CZE & 4 & 0,178 & 97,243 \\
\hline DNK & 4 & 0,178 & 97,421 \\
\hline BGR & 3 & 0,133 & 97,554 \\
\hline HUN & 3 & 0,133 & 97,688 \\
\hline SWE & 3 & 0,133 & 97,821 \\
\hline Australie & 2 & 0,089 & 97,91 \\
\hline Autriche & 2 & 0,089 & 97,999 \\
\hline PRT & 2 & 0,089 & 98,088 \\
\hline ESC & 2 & 0,089 & 98,177 \\
\hline
\end{tabular}




\begin{tabular}{|l|l|l|l|}
\hline Argentine & 1 & 0,044 & 98,221 \\
\hline CHE & 1 & 0,044 & 98,265 \\
\hline Chili & 1 & 0,044 & 98,309 \\
\hline Hollande & 1 & 0,044 & 98,353 \\
\hline IRL & 1 & 0,044 & 98,397 \\
\hline ISR & 1 & 0,044 & 98,441 \\
\hline NZL & 1 & 0,044 & 98,485 \\
\hline SVK & 1 & 0,044 & 98,529 \\
\hline THA & 1 & 0,044 & 98,573 \\
\hline Yougoslavie & 1 & 0,044 & 98,617 \\
\hline NR & 34 & 1,512 & 100 \\
\hline Total & 2249 & 100 & \\
\hline
\end{tabular}

Source: base SNI, 2009.

\section{NOTES}

1. Ces enseignements sont "naturellement" partiels, dans la mesure où la base ne concerne que $10 \%$ environ de l'ensemble des chercheurs et enseignants chercheurs, et uniquement ceux qui exercent dans leur pays. La base réfère donc à des populations mobiles durant leurs études, non aux "migrants scientifiques".

2. 66 entretiens ont été réalisés dans diverses institutions du district fédéral (UAM, UNAM) et d'autres Etats (Aguascalientes, Nuevo Léon, Jalisco, Sonora).

3. Dans la seconde moitié du vingtième siècle, elles faisaient partie des dix carrières les plus demandées au Mexique (source : ANUIES, 2012).

4. Les instituts publics comptent 79,7\% des étudiants, les universités privées, 20,3\% (ANUIES, 2012).

5. La population des chercheurs-ingénieurs membres du SNI représentait, en $2009,14,9 \%$ du total des chercheurs de ce système. Cette population est très masculine. Alors que la proportion de femmes, au niveau de la licence et aux deuxième et troisième cycles, est globalement (toutes disciplines) quasiment égale à celle des hommes (respectivement 49,8\% et $49 \%$ ), elle représente moins d'un tiers dans les disciplines des sciences de l'ingénierie (30,3\% en licence et $32,1 \%$ pour les cycles supérieurs).

6. Les autres domaines disciplinaires et leurs proportions de chercheurs formés à l'étranger sont : 1) la physique, les mathématiques et les sciences de la terre $(37,6 \%), 2)$ la biologie et la chimie $(22,8 \%), 3)$ la médecine et les sciences de la santé $(14,3 \%), 4)$ les humanités et sciences du comportement $(29,7 \%), 5)$ les sciences sociales $(40,8 \%), 6)$ la biotechnologie et les sciences agrovétérinaires (43,6\%). Source : Didou et Gérard, op. cit., p. 61. 
7. Ce taux correspond à la proportion d'étudiants inscrits dans l'enseignement supérieur par rapport à la population scolarisable en âge de suivre ce degré d'enseignement (20-24 ans).

8. Padrón Nacional de Posgrado de Calidad. Au Mexique, existent de nombreux systèmes de bourses nationaux et étrangers. Au niveau national, sont octroyées des bourses par le CONACYT et le Ministère de l'éducation, qui soutient les enseignants chercheurs pour la réalisation de leur maîtrise et doctorat. Les bourses du CONACYT pour étudier au Mexique sont, notamment, accordées aux Programmes de posgrado (maestria, especialización et doctorado) reconnus par ce PNPC. Grediaga Kuri note que, en 2010, 44,75\% des programmes de doctorat étaient ainsi reconnus et, donc, éligibles pour les bourses CONACYT (Grediaga, 2012). Enfin, les bourses CONACYT pour étudier à l'étranger sont accordées aux étudiants selon la position de leur institution dans la hiérarchie du Web of Science.

9. En matière d'ingénierie notamment, le Mexique et la France collaborent autour de deux programmes, depuis 2001 et 2008 respectivement: le «Programme de coopération dans le domaine des formations technologiques et professionnelles de l'enseignement supérieur» (Programa de Cooperación en el Área de las Formaciones Tecnológicas y Profesionales de la Enseñanza Superior) et le programme Mexprotec ("Seguimiento ex becarios del programa de movilidad con Francia”, Subsecretaria de Educacion superior, coordinacion general de Universidades). Ces deux programmes ont permis à 280 Techniciens supérieurs universitaires boursiers (Técnicos Superiores Universitarios), de 30 universités technologiques (Universidades Tecnológicas) de bénéficier de formations en France.

10. Dans le cas des étudiants mexicains en France, notamment, les données de Campus France révèlent que $21,2 \%$ d'entre eux, en moyenne sur la période 2006-2012, étaient boursiers (la proportion la plus basse ayant été de $13,9 \%$ en 2007 , la plus haute, de $32,7 \%$ en 2012).

\section{RÉSUMÉS}

Ce travail se propose d'examiner l'héritage de traditions scientifiques dans l'ingénierie mexicaine, par l'analyse des «pôles internationaux» de formation des ingénieurs. Nous montrons que, à l'instar des autres disciplines, la formation en ingénierie s'endogénéise en quelque sorte au Mexique : la part des chercheurs formés à l'étranger diminue de génération en génération. Cependant, des liens historiques entre des pays occidentaux (Etats-Unis, France, Angleterre) et le Mexique perdurent, ainsi que des relations étroites entre des institutions de formation mexicaines et étrangères. Ces relations ont les traits de "chaînes de savoirs", maintenues grâce aux trajectoires successives des élèves-ingénieurs. À l'examen, ces chaînes semblent dépendre de la structuration du champ scientifique étranger. Ainsi, les mobilités pour études sont davantage "instituées" vers des pays européens que vers les Etats-Unis. Et ils diffèrent d'une sous-discipline de l'ingénierie à une autre. En complément de données statistiques tirées de la base du Système national des chercheurs (SNI), des entretiens qualitatifs illustrent l'absence de reproduction simple des trajectoires de formation et leur complexité. Mis en regard, ces deux ensembles de données conduisent à montrer que les trajectoires individuelles sont "enchâssées" dans des logiques sociales et politiques de mobilité et de formation, ou encore de division internationale du travail scientifique. Des logiques qui, peu à peu et pour certains pôles traditionnels de formation, remettent en cause les "héritages" scientifiques des générations passées. 
Este trabajo se propone analizar la herencia de tradiciones científicas en el campo de la ingeniería Mexicana. Se muestra que, al igual que en otras áreas disciplinarias, la formación en ingeniería se vuelve, en algún sentido, cada vez más endogámica: la proporción de ingenieros formados en el extranjero respecto a quienes se forman en México disminuye de generación en generación. No obstante, los lazos históricos entre los países occidentales (Estados Unidos, Francia, Inglaterra) y México perduran como relaciones estrechas entre las instituciones mexicanas y las instituciones extranjeras de formación. Estas relaciones tienen rasgos de "cadenas de conocimiento", que se mantienen gracias a las trayectorias formativas de las generaciones sucesivas de los estudiantes de ingeniería. Al examinarlas, parecería que la continuidad de estas cadenas depende en gran medida de la estructuración del campo científico internacional. Si bien las movilidades están "institucionalizadas" históricamente, ya que a través del tiempo han estado más vinculadas con Europa que con Estados Unidos, esta institucionalización es variable de una sub disciplina a otra. A partir de los datos tomados del Sistema Nacional de Investigadores (SNI) y de entrevistas a profundidad se ilustra que no se puede hablar de reproducción simple de las trayectorias formativas. La comparación de los datos entre generaciones muestra que las trayectorias individuales están ligadas cada vez más a lógicas sociales, las políticas de movilidad y apoyo a la formación, o más aún, a la división del trabajo científico en el contexto internacional. Estas lógicas ponen en cuestión la reproducción de la orientación respecto a ciertos polos tradicionales de formación, es decir, la reproducción simple o herencia científica de las nuevas generaciones de los caminos seguidos por las generaciones pasadas.

This paper proposes to examine the legacy of scientific traditions in Mexican engineering by analyzing fluxes between Mexico and the international centers of engineering education. We show that, like other disciplines, training in engineering is somehow increasingly endogenous in Mexico: the engineer researchers trained abroad decline from generation to generation. However, the historical ties between Western countries (USA, France, England) and Mexico remain, as do the close relations between Mexican and foreign training institutions. These relationships have traits of "knowledge chains", maintained through successive exchanges of students from different generations of Mexican engineers. Thus, mobility for studies in this field are more "institutionalized" or historicized to European countries than to the United States. Of course differences prevail from one sub-discipline of engineering to another. With the statistical data analysis, extracted from the database of the National System of Researchers (SNI), supported by in-depth interviews, we cannot speak of simple reproduction patterns of training trajectories, but of an increasing complexity of training circuits. The comparison of these two data sets lead us to comprehend how individual trajectories are "embedded" in social and political logics of mobility and training or the international division of scientific work. The analysis between generations shows that individual trajectories are increasingly associated with social and political mobility and training logics, or even more so, to the scientific international division of labor. These trajectories challenge, in certain areas of specialization, the tradition of scientific heritage following the paths of previous generations.

\section{INDEX}

Mots-clés : Mexique, ingénierie, mobilité étudiante et académique, pôles de formation, chaînes de savoir

Keywords : Mexico, engineering, student and scholars' mobility, chains of knowledge

Palabras claves : México, ciencias de la tecnología, movilidad estudiantil y académica, polos de formación, cadenas de saber 


\section{AUTEURS}

\section{ETIENNE GÉRARD}

Sociologue, directeur de recherches, Institut de recherche pour le développement, UMR Ceped

(Université Paris Descartes, IRD, Paris. etienne.gerardatird.fr

\section{ROCIO GREDIAGA}

Sociologue, Professora de tiempo completo, UAM Azcapotzalco, Mexico.

mrgkatcorreo.azc.uam.mx 